\title{
Begin boulevard in Tel Aviv - from a suburban highway to an urban boulevard
}

\author{
Y. Rofè ${ }^{1} \&$ R. Ishaq ${ }^{2}$ \\ ${ }^{1}$ Blaustein Institutes of Desert Research, \\ Ben-Gurion University of Negev, Israel \\ ${ }^{2} P G L-$ Perlstein Galit Ltd, Israel
}

\begin{abstract}
Tel Aviv's Central Business District (CBD) is centered around Begin Road. By 2020 , this area is expected to have an increase of $50 \%$ in employment and $40 \%$ in housing.

Initial proposals for Begin Road sought to meet the increased demand for travel by increasing road capacity. In response, the Israel Ministry of Transport mandated an alternative design, which would give priority to public transit and pedestrians. The requirements of the alternative plan were: create a street with positive and clear urban identity, provide accessibility and safety for pedestrians and bicycles, give real priority for public transportation, allow through-traffic and provide access to street frontages, including short term parking.

Five alternatives were developed by the planning team. All of them were different versions of the "multi-way boulevard". The "multi-way boulevard" creates a clear separation between a slow moving "pedestrian realm", and fast movement in the "through-traffic realm." A multi-disciplinary evaluation process, involving professionals from several disciplines, was used to select two alternatives for further study. Evaluators first ranked the different road uses. Then each design was evaluated to provide a score on the overall integration and performance for the road design.

Despite differences in priority schemes given to bus transit, both of the preferred alternatives provide a good balance between through traffic, vehicular access to adjacent uses, public transportation, and pedestrian and bicycle movement. They also create a strong, memorable street attractive to its users, which will encourage new development in the area.
\end{abstract}

Keywords: boulevards, main streets - design, transportation planning, traffic engineering, public transit, pedestrian movement. 


\section{Introduction}

In recent years transportation planning and traffic engineering have been under severe criticism [5, 6], undergoing far ranging modifications in their basic principles and methods. In the design of residential areas it has brought the return to traditional grid-based street design [2], combined with traffic calming techniques [7] to slow cars down and reduce the hazards of vehicles for pedestrians. The design of major urban streets has brought, among other approaches, the return of the urban boulevard [4], as a form of street that can handle both high volumes of traffic, as well as public transit, pedestrian and bicycle movement, access to buildings and a vibrant urban life.

This paper presents the design process for Begin Road, a thoroughfare that is evolving as the main artery of the CBD of Tel-Aviv Metropolitan Area. The design approach was based on the concept of the Multi-Way Boulevard, under which several alternative designs were developed. This new planning paradigm, where vehicular capacity no longer dominates, is based on a more complex understanding of the urban street.

The act of designing major urban streets has become an act of balancing between several, possibly conflicting, but mutually supportive requirements. This balance can be achieved in different ways, depending on the urban context, the intentions of policy makers, and the dynamics of the area. It is therefore important that an open and clear method is devised for evaluating all alternatives. The method has to allow the various requirements to be ranked, and their impact on the design evaluated. This method has to be applied throughout the process: as part of design stage, in order to distinguish between alternatives, and to select the more promising alternatives for further design development; and finally in the decision making process, selection of a preferred alternatives and the development of the final design. This paper describes the design process up to the selection of the two alternatives for further design development.

\section{The need for a change in paradigm: Begin Road and its changing urban context}

\subsection{Changes in land-use and building volumes}

The City of Tel Aviv serves as the main employment center for the Tel Aviv Metropolitan Area, and the hub of economic and cultural activities in Israel. Despite the suburbanization of employment and commercial activities over the last few years, the Tel Aviv Metropolitan CBD has kept its dominance in employment, particularly in the financial services sector.

The continued development of the Metropolitan CBD is based on the District Outline Plan No. 5, currently under review for approval. This plan designates high-rise office buildings along Begin Road, allowing for an increase of 50\% in the number of employees (from 60,000 in 2000 , to 90,000 in 2020 ), and of $40 \%$ in residential housing (from 30,500 in 2000 , to 42,500 in 2020). 


\subsection{Changes in the transportation system}

Begin Road, previously named Petah-Tikva, is a thoroughfare in the heart of the Metropolitan CBD. It is part of the historical road that has connected Jaffa and Tel-Aviv to the first northeastern suburbs of Ramat-Gan, Bnei-Brak and PetahTikvah. At the north end of the project area, it is joined by Namir Road, which connects the CBD with Tel-Aviv's northern neighborhoods and suburbs.

Until the construction of the "Ayalon Freeway" in the early 1970s, PetahTikva Road served as the major axis for inter-urban trips. The construction of a freeway in parallel to this road has shifted most of those trips toward the freeway. Today, Begin Road is the main thoroughfare for trips with destination in the $\mathrm{CBD}$, and for some of the through traffic to nearby areas. According to the 1995 Population Census Survey, about 50\% of the employees traveled to Begin Road by car, $34 \%$ by bus and only $16 \%$ by other means (train, walk and bicycle). Since 1995, with improved train frequency and level of service, trips by rail to the area have increased. However, the majority of the trips remain by car and bus.

Bus service plays an important role in moving employees to the CBD includes urban, metropolitan and inter-urban routes. Along Begin Road bus volume, reaches 4,000 buses per day, and two major inter-urban bus terminals the Tel-Aviv "Central Bus Station" and "Terminal 2000" - are located at each end of the project area.

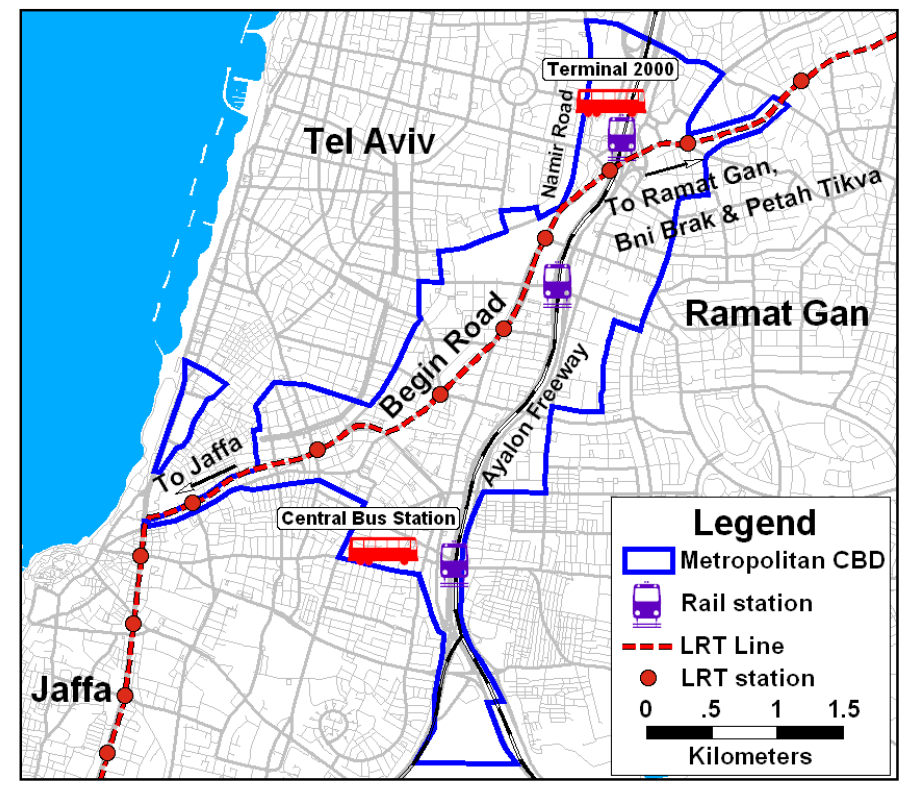

Figure 1: Begin Road in the context of the Metropolitan CBD. 
Current traffic conditions as well as the inability of the current road system to support accessibility needs of future development, led the Ministry of Transport (MOT) to change its policy regarding transport and traffic in the CBD. This policy shifts the emphasis from the private automobile to public transit and alternative modes of travel. It designates six components to achieve a change in modal split:

1. Continuing development of the Suburban Rail system running within the Ayalon Freeway.

2. Constructing a Metropolitan Light Rail System (LRT). The first LRT line will operate underground along Begin Road (scheduled to open in 2013).

3. Developing metropolitan bus re-organization plan with more hierarchical and coherent system.

4. Implementing new parking standards, which limit parking provision in areas well served by public transit.

5. Implementing high occupancy toll (HOT) lanes on the Ayalon Freeway approaches to the CBD.

6. Providing priority access for pedestrians and bicycles in the CBD.

\subsection{Change in urban role: from metropolitan thoroughfare to the main street of the emerging metropolitan CBD}

Changes in land use and transportation caused a change in the urban role played by Begin Road. Originally, an arterial road, conducting intra-urban traffic and lined with light industry, mechanical repair shops, the wholesale market and dairies, it is being transformed into the main street of the emerging CBD. It is also becoming the gateway to the transforming historic city, the cultural and entertainment center.

\section{Design alternatives for Begin Road}

\subsection{Initial design by the Netivei Ayalon Highway Company}

At the request of the Municipality of Tel-Aviv, and the District Planning Commission, the Netivei Ayalon Highway Company (NAHC), a government company, owned jointly by the MOT and the Tel-Aviv Municipality, prepared an initial, comprehensive plan for Begin Road. This plan failed to recognize the change in the Ministry's priorities and concentrated on expediting the movement of private vehicles. The plan met with criticism by NTA, the company developing the LRT system, the Public Transit Administration within NAHC, and from other MOT officials. It created rift within the municipality, where the advocacy for pedestrians and bicycles is growing, in contrast to the prevailing policy of increasing road capacity and vehicular access, as basis for continued development of the CBD. 


\subsection{A counter proposal - Begin Road redesigned as a boulevard}

As a reaction to the initial plan proposed by NAHC, the MOT asked our Study Team to develop alternative designs, to demonstrate the impacts of shifting from dominance of automobile traffic to a more balanced approach, which accommodates all users of the street. This new approach is expressed in the following design requirements:

1. Establishing an urban street of quality and identity which provides a positive urban context for growth

This requirement is a function of the changing role of Begin Road. The road is destined to be the major artery of Tel-Aviv CBD in the 21 st century. The process of growth of the CBD is gradual, and the transformation of land uses along the street is expected to be slow. Thus the development of the street as a pleasant, coherent, and tree-lined boulevard will create a location with identity and quality, which will help to attract development to the area.

2. Priority for pedestrian movement, comfort and safety

Pedestrian movement is expected to increase, especially after the LRT becomes operational. It will include all public transit passengers, for whom Begin Road is their final destination, and future residents working in the immediate area. Pedestrian needs include safe and comfortable sidewalks and crossings, as well as an active street frontage with shops and cafés, which makes walking more interesting and provides opportunities for social activity [1].

3. Promoting bus transit.

The future operation of the LRT is expected to somewhat reduce the number of bus patrons. Yet, analysis has shown that this reduction will be limited to only about $16 \%$ of bus ridership today. This, combined with the planned development along the street, indicates that the number of bus riders to the CBD may actually increase. Thus, there is a need to provide exclusive bus lanes along street.

4. Bicycle lanes

Creation of safe and continuous lanes for bicycles that will enhance the bicycle network developed by the city of Tel-Aviv.

5. Providing short term parking

A road with active frontage of shops and building entrances will inevitably need parking space for deliveries, and for store patrons. If short-term parking is not legally provided, it tends to happen illegally, by parking on the curb lane, or on the sidewalks.

6. Maintaining through traffic

Even though today Begin Road is largely a local distributor to the CBD, it still significant handles amount of through-traffic to the old city center, and between south and north Tel-Aviv.

\subsection{The Boulevard concept and the "Pedestrian Realm"}

Boulevards are streets that were perfected in the 19th century to deal with the growing complexity of the city and its streets. Today they still function as major main streets in many cities, as shown by Jacobs and colleagues [3, 4]. They describe three types of boulevards: the center median boulevard, the boulevard 
street, and the multi-way boulevard. The center median boulevard is distinguished by its wide central median flanked by roadways. It is most appropriate as a pedestrian promenade like the Ramblas of Barcelona, or as in a local example of Rothschild Boulevard in Tel Aviv. The boulevard street is similar in its cross-section to ordinary streets, but is usually wider and more accommodating in its sidewalks, and includes the characteristic row of trees separating the sidewalk from the roadway. The multi-way boulevard was found by Jacobs et al. to hold particular potential for resolving the many contrasting requirements that a major urban street needs to serve. Since the first type was deemed inappropriate, because of the amount of vehicular traffic that the road still has to serve, only designs of the second and third types were considered.

Central to the success and safety of multi-way boulevards described by Jacobs and his colleagues [4] is the concept of "the pedestrian realm." This is an area, where vehicles may enter, but which is essentially at the pedestrian scale and speed. It allows for walking, staying, access and social activities. It was observed that in all successful multi-way boulevards this realm, extending from the flanks of both sides of the street, occupied at least $50 \%$ of the street's crosssection. It was also separated from the central thoroughgoing realm by a strong and continuous row of trees. In the following design alternatives, particular attention was paid to the creation of such a "pedestrian realm."

\section{4 "Begin Boulevard" - design alternatives}

The initial alternative designs developed by the Study Team were the two basic boulevard cross-sections: 1) a street boulevard, and 2) a multi-way boulevard. As the project progressed, three additional alternatives were considered: 3) a multi-way boulevard with exclusive bus-ways in the side lanes, 4) a refinement of alternative 3 , which included short term parking on the central thoroughfare and bicycle lanes on the medians, and 5) an exclusive central bus-way boulevard. A typical cross-section was created for each alternative for a 44-meter wide right of way - the most prevalent right of way along Begin Road.

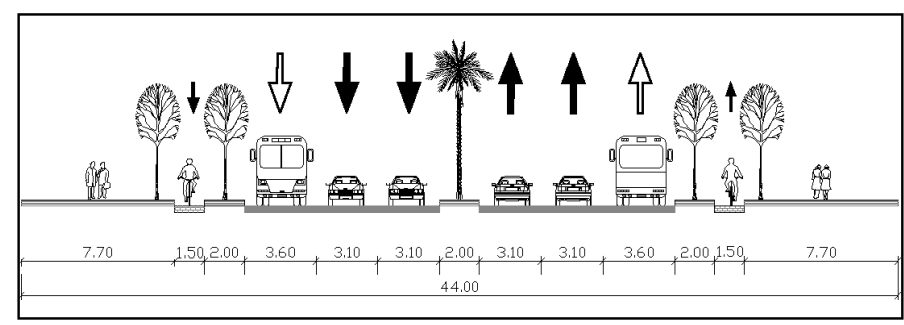

Figure 2: A street boulevard typical cross-section.

\subsubsection{Alternative 1: a street boulevard}

The boulevard contains a "pedestrian realm" which is typically 11.20 meters $(7.70+1.50+2.0)$ on each side. It contains a wide sidewalk for pedestrians, and a bicycle lane between two rows of trees that separate the pedestrian realm from 
the thoroughgoing traffic area. The traffic facility contains a designated bus lane, and two lanes for private vehicles. The major drawback of this kind of boulevard is that it does not allow for short-term parking, and therefore there is a risk of blockage of the bus lane by illegal stopping, parking and delivery vehicles.

\subsubsection{Alternative 2: a multi-way boulevard}

The "pedestrian realm" of the multi-way boulevard contains within it the sidewalk and a service street. This street is planned according to traffic calming standards, recently approved in Israel. Its reduced width and many interruptions allow only very slow car movement. It is therefore possible to include within it bicycles and it provides convenient pedestrian crossing to the bus stops on the medians. The thoroughgoing traffic lanes have a designated right-hand side bus lane, and two lanes for private vehicles. In this case disruption of the bus lanes is minimized, because the access street allows for short-term parking and deliveries.

Officials at the MOT felt that the multi-way boulevard did not give enough priority to the heavy bus volumes expected on Begin Road. Thus, they asked the Study Team to develop additional alternatives that provide exclusive and separate right of way for buses. The three alternatives are shown below.

\subsubsection{Alternative 3: a multi-way boulevard with curb-side busways}

The pedestrian realm in alternative 3 contains the sidewalk and a bicycle lane. The exclusive bus-way creates additional separation between the pedestrians and fast moving through traffic in the center lanes. Planning guidelines stipulate a width of 5.40 meters for such facilities, to allow for overtaking of a stalled bus. However, it was felt that such width would encourage excessive speeds on the busways, endangering pedestrians and impacting negatively the pedestrian realm. Therefore, the busway was planned as 3.6 meters wide and a 1.8 meters wide sloping "skirt", free from obstacles, was designed on the traffic island. The design still allows overtaking in an emergency, but will not encourage regular use. This boulevard design does not supply short-term parking. Therefore, there is a risk of interruption by illegally parked vehicles.

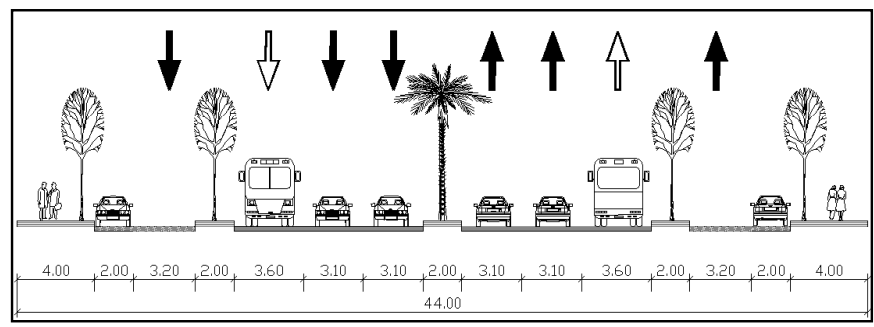

Figure 3: A multi-way boulevard typical cross-section. 


\subsubsection{Alternative 4: a multi-way boulevard with curb-side busways}

This cross-section represents a variation of alternative 3. By shifting the bicycle lane to the median island, it also serves as the emergency "skirt" for the busway. This reserves space for wide sidewalks and for short-term parking adjacent to the central lanes. The pedestrian realm contains the sidewalk, an exclusive busway (3.6 meter wide) and a bicycle lane (1.5 meter wide). In case of emergency, buses can utilize the bicycle lane to pass a stalled bus. The central lanes include two thoroughgoing traffic lanes and a short term parking lane.

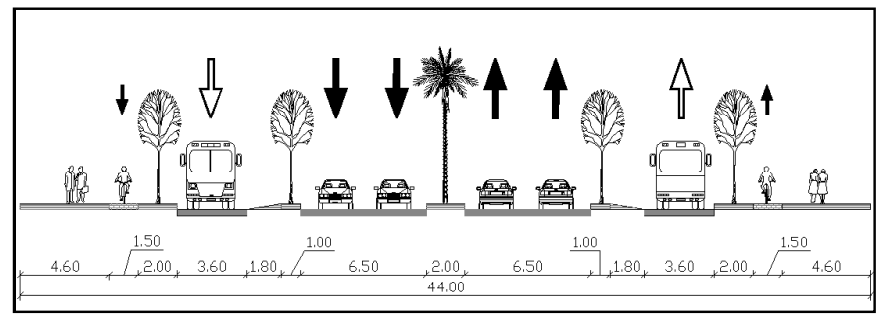

Figure 4: A multi-way boulevard with side bus-way typical cross-section.

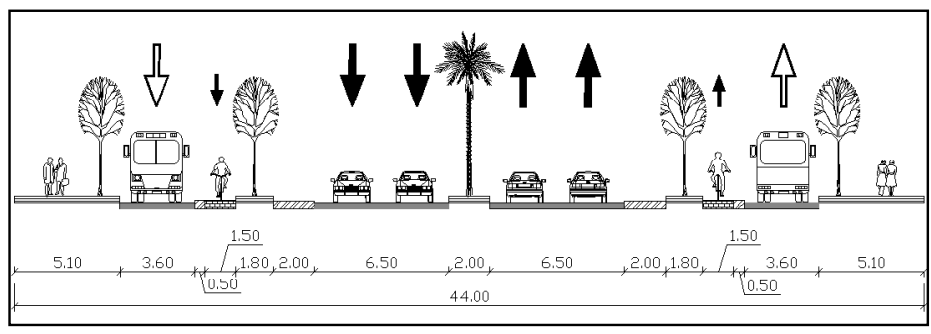

Figure 5: A multi-way boulevard with curb-side busway: typical crosssection.

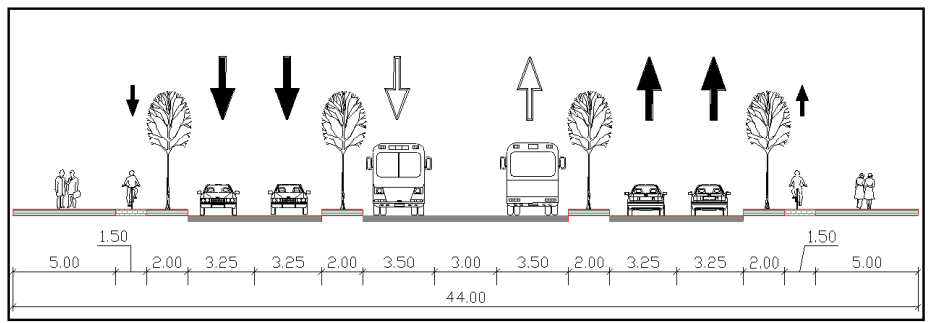

Figure 6: A central bus-way boulevard typical cross-section. 


\subsubsection{Alternative 5: boulevard with a central busway}

Finally, the Study Team examined a cross-section, which provides the highest priority to bus traffic. The "pedestrian realm" in this kind of boulevard, contains a sidewalk and a bicycle lane. The thoroughgoing traffic area contains two lanes for vehicles and a centrally located exclusive busway. This can be seen as a variation of the central island boulevard, where instead of a central promenade there is a busway. Experience shows that it works well when the private traffic lanes are narrow and serve only access traffic. Otherwise, crossing from the sidewalk to the bus stops on the medians across two lanes of moving traffic is dangerous to pedestrians. This kind of boulevard, does not provide short term parking, and while illegally stopped and parked cars on one of the through lanes may decrease vehicle speed and improve pedestrian safety, they may also grossly reduce the street's capacity to handle through traffic.

\subsection{The evaluation process}

Evaluation of the five alternatives revealed the strengths of each design. The first alternative provides the widest sidewalks and the simplest intersections. The second allows for an elegant solution to access to frontage uses. The last three alternatives provide an increased priority for buses. However, none of them resolves in an optimal way all of the requirements. The Study Team developed an evaluation process to select the prefered alternative. It was first used as a design tool to choose the two preferred cross-sections for further development, leading to a detailed design. The Study Team assembled a group of ten professionals of various disciplines: road engineering, traffic engineering, transportation planning, public transportation planning and urban design. A typical cross-section and a plan layout of a street section with two intersections were presented to each of the participants. They were first asked to evaluate how the design met the needs of different road users. In addition, they were asked to weigh the relative importance of each usage as it contributed to the overall street performance. The results of the evaluation process are presented in Table 1, and the grades assigned to each alternative in Figure 7.

Table 1: Weights for meeting each requirement.

\begin{tabular}{|l|c|}
\hline \multicolumn{1}{|c|}{ Criteria } & Weight \\
\hline Establishing an urban street of quality and identity & $17 \%$ \\
\hline Priority for pedestrian movement, comfort and safety & $23 \%$ \\
\hline Promoting bus transit & $22 \%$ \\
\hline Bicycle lanes & $13 \%$ \\
\hline Providing short term parking & $10 \%$ \\
\hline Facilitating through car traffic & $15 \%$ \\
\hline Total & $\mathbf{1 0 0 \%}$ \\
\hline
\end{tabular}




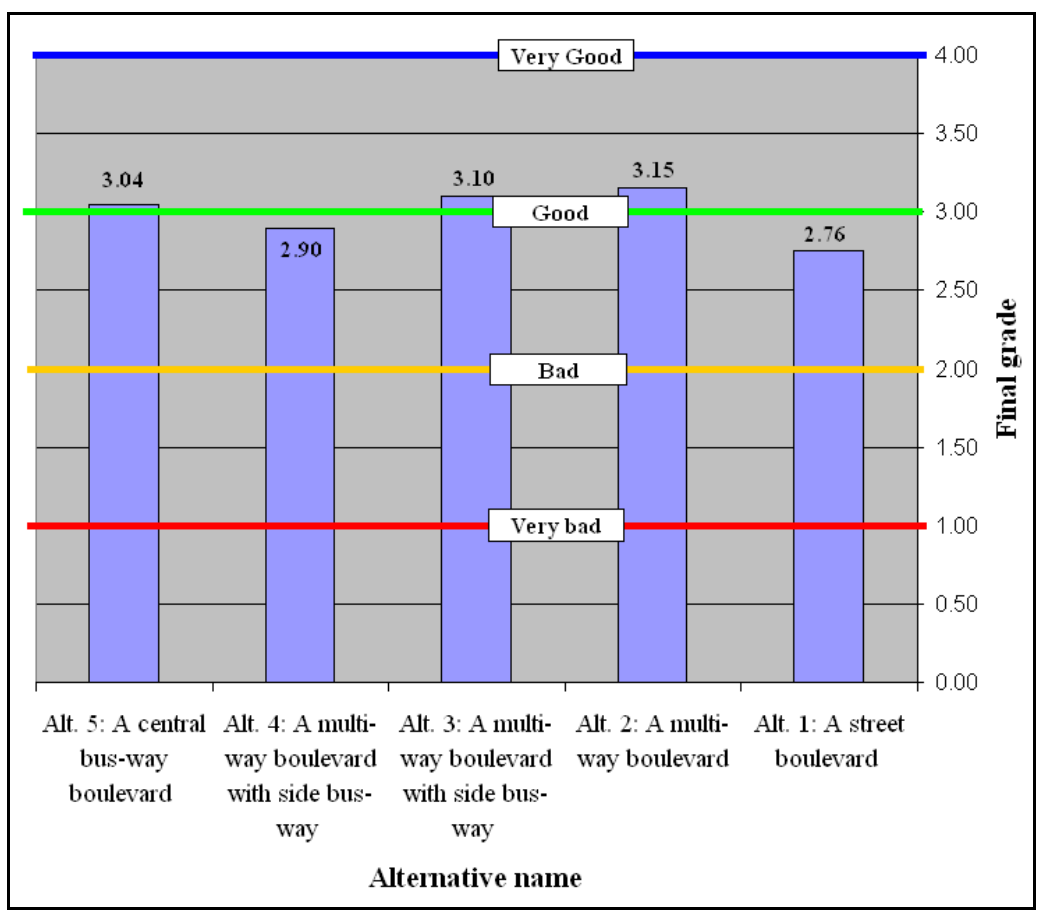

Figure 7: Grades for each alternative.

In general, all alternatives achieved scores that placed the design in an acceptable range. However, all of the multi-way boulevard sections score better than the street boulevard section (alt. 1). Out of five alternatives, three passed the score of 3 (overall assessment as a "good" fit to the requirements). Two alternatives that were chosen for design development are the "multi-way boulevard"(alt. 2) and the "multi-way boulevard, including exclusive right-hand side busway and short term parking" (alt. 4). Alt. 4 was selected, even though it received a slightly lower score than the "exclusive right-hand busway boulevard" (alt. 3). This is because unlike the former, it included short-term parking, and thus met all of the requirements.

\section{Conclusion: developing a new paradigm for major urban streets}

The need for a changed paradigm in the design of Begin Road evolved, as it became clear that private automobile could not meet the accessibility needs of the CBD. However, making a change toward public-transit and pedestrian oriented environment is not completed until it incorporates appropriate street designs. The way main streets are designed needs to shift from considerations of vehicular capacity and speed, to that of a complex space catering to many needs and requirements. These requirements refer to six different components: 
establishing an urban street with identity and quality, priority for pedestrians, promoting public transit, allowing continuous and safe bicycle lanes, providing short term parking opportunities, and doing all of that while maintaining through traffic. A means of responding to these needs is the "multi-way boulevard". The cross-section of a "multi-way boulevard" allows the coexistence and interaction between all travel modes, movements and uses. The establishment of a slow moving "pedestrian realm," and its separation from a faster moving "thoroughgoing realm" by the consistent row of trees is the hallmark of boulevards, which gives the street form its high performance and safety. Under this principle, many variations are possible, depending on the relative importance given to each requirement.

In the case of Begin Road, five alternative designs were examined. All of them have proven more versatile and responsive to the requirements than the original car oriented design. However, they differed in the allocation of space for each use and in the way they met the design requirements. Each design set a different balance between pedestrian movement, bus transit, and access to street frontages. The evaluation process made it clear that the selection was not based on some objectively defined criteria, but rather depended on the conception of the street as a whole, and what was deemed more important within it. The two road sections selected for further elaboration were two versions of the "multiway boulevard", one providing service roads with short-term parking, and the other using the side ways for dedicated bus lanes and bicycles.

\section{Acknowledgements}

The Ayalon Highway Company, Department of Public Transportation, under the management of Ruti Amir sponsored this work on behalf of the Ministry of Transport. The authors thank the rest of the PGL project team - Rebecca Sliselberg, Tatyana Smiller, Roni Adiv and Marcos Szeinuk.

\section{References}

[1] Gehl, J. Life Between Buildings, Arkitektens Forlag: Copenhagen, 1996.

[2] Hebbert, M., New Urbanism: the movement in context. Built Environment, 29(3), pp. 193-209.

[3] Jacobs, A.B., Great Streets, MIT Press: Cambridge, Mass. 1994.

[4] Jacobs, A.B, Macdonald, E. and Rofè, Y., The Boulevard Book, MIT Press: Cambridge, Mass., 2002.

[5] Marshall, S. Streets and Patterns, Spon Press: London and New York, pp. 1-19, 2005.

[6] Murrain, P., Understand urbanism and get off its back, Urban Design International, 7, pp. 131-142, 2002.

[7] Pucher, J., Dijkstra, L. Making walking and cycling safer: lessons from Europe, Transportation Quarterly 54(3), pp. 25-50, 2000.

[8] Southworth, M. and Ben-Joseph, E., Streets and the Shaping of Towns and Cities, McGraw-Hill: New York, 1997. 